\title{
Effect of a nonlinear refractive index on Faraday rotation
}

\author{
Yi Jung $\mathrm{Yu}^{*}$ and Richard K. Osborn \\ Department of Nuclear Engineering, University of Michigan, Ann Arbor, Michigan 48105 \\ (Received 1 June 1976; revised manuscript received 26 January 1977)
}

\begin{abstract}
A theory for the nonlinear (with respect to the electric field) Faraday effect has been developed and applied to a simple model. Within the framework of this model, our analysis shows that the ratio of the nonlinear Verdet constant to the linear Verdet constant is proportional to the intensity of the incident light and depends on the frequency of light, the excitation energy of the model molecule, and the temperature of the medium. This ratio is small under ordinary conditions. However, under certain circumstances it may become large enough for experimental observation.
\end{abstract}

\section{INTRODUCTION}

In 1845 Faraday $^{1}$ demonstrated that the polarization plane of linearly polarized light could be rotated after passing through certain media which were placed in a magnetic field. Since then, the interest in the Faraday effect has stimulated much experimental and theoretical research. ${ }^{2}$ But the study is limited to the linear (with respect to the electric field) effect. The phenomenon is described by a simple formula, $\theta=V L \overrightarrow{\mathrm{B}} \cdot \hat{K}$, where $\theta$ is the angle of rotation of the polarization plane of the incident, linearly polarized light, $L$ is the thickness of the Faraday medium, $\vec{B}$ is the applied magnetic field, and $\hat{K}$ is the unit wave vector of the incident light. $V$ is the Verdet constant, which depends on the frequency of the incident light, the microscopic structure of the medium, and the temperature of the medium. More explicitly, $V$ is proportional to the square of the frequency of the incident light, and is separable into two parts: a diamagnetic part which is independent of the temperature, and a paramagnetic part which is inversely proportional to the temperature.

Recently, because of the upsurge of interest in high-power laser technology, ${ }^{3,4}$ the nonlinear response of various media to intense light beams has received much attention. One scheme for the operation of a laser system is to use Faraday rotators to protect the laser from target-reflected energy. Furthermore, in some laser systems, ${ }^{3}$ large aperture Faraday rotators have been introduced into regions of high beam intensity.

The theory and experimental status of the linear Faraday effect is summarized by Van Vleck. ${ }^{2}$ An extensive treatment of this effect is given by Rosenfeld. ${ }^{5}$ Application of the theory for rare-earth ions and molecules has been examined by Serber ${ }^{6}$ and by Van Vleck and Hebb. ${ }^{7}$ However, the nonlinear (i.e., intensity-dependent) Faraday effect has not been studied. Thus, the purpose of the work described in the present paper will be threefold. First, we will develop a theory for the inten- sity-dependent Faraday effect. Second, a simple model will be used to illustrate its qualitative features. Third, we will correlate the linear and nonlinear Faraday effect, and establish an empirical relationship between the two as a guide for experimental study. The latter is analogous in spirit to Wang's ${ }^{8}$ study of optical susceptibilities, but the content is quite different.

It will be shown in the context of the simple model that the nonlinear Verdet constant is proportional to the intensity of the light. Furthermore, it is found that the frequency dependence of the nonlinear Verdet constant is quite different from that of the linear Verdet constant. Most strikingly, we find a term inversely proportional to the square of the temperature in the paramagnetic part of the nonlinear Verdet constant. Possible implications, applications, and extensions will be given in the discussion section.

In what follows we will divide the paper into three sections. The first section will be devoted to the development of the general theory and a brief review of the linear Faraday effect. The reason for rederiving the general theory-instead of using results presented elsewhere ${ }^{9,10}$-is that we wish to make use of simplifications due to modeling at intermediate points in the analysis. In the second section a simple model will be used to facilitate the derivation of explicit formulas for the linear and nonlinear Faraday effect. In the last section an empirical relationship between the linear and nonlinear effect and a discussion of possible implications will be presented.

\section{GENERAL THEORY}

The angle of rotation $\theta$ is related to the index of refraction through

$$
\theta=(\omega / 2 c)\left(\eta_{+}-\eta_{-}\right) L
$$

where $\omega$ is the angular frequency of the incident light, and $\eta_{+}$and $\eta_{-}$are indices of refraction for the right and the left circularly polarized light, 
respectively. The index of refraction $\eta$ is related to the electric susceptibility $\chi$ which in turn is related to the polarization density $\langle\overrightarrow{\mathrm{P}}\rangle$. The calculation of $\langle\overrightarrow{\mathrm{P}}\rangle$ will be carried out by conventional successive approximation..$^{9,10}$ A formula for $\langle\overrightarrow{\mathrm{P}}\rangle$ is

$$
\langle\overrightarrow{\mathrm{P}}\rangle=\operatorname{trace}(N \vec{\mu} D)
$$

where $N$ is the number density of the molecules in the medium, $\vec{\mu}$ is the dipole moment operator for a molecule, and $D$ is the probability operator. The probability operator satisfies the Liouville equation

$$
\frac{\partial}{\partial t} D=\frac{i}{\hbar}[D, H]
$$

where $H=H_{0}-\vec{\mu} \cdot \overrightarrow{\mathrm{E}}(t)$ is the Hamiltonian of a single molecule, $H_{0}$ is the unperturbed part, and $\overrightarrow{\mathrm{E}}(t)$ is the electric field associated with the incident light. The calculation of $\langle\overrightarrow{\mathrm{P}}\rangle$ yields

$$
\langle\overrightarrow{\mathrm{P}}\rangle=\left\langle\overrightarrow{\mathrm{P}}^{1}\right\rangle+\left\langle\overrightarrow{\mathrm{P}}^{2}\right\rangle+\left\langle\overrightarrow{\mathrm{P}}^{3}\right\rangle+\cdots
$$

where the upper indices indicate the order of approximation. As discussed in Ref. 9, $\left\langle\overrightarrow{\mathbf{p}}^{2}\right\rangle$ gives rise to dc terms and second harmonic generation, and $\left\langle\overrightarrow{\mathbf{P}}^{3}\right\rangle$ contains terms which vary with time like $\overrightarrow{\mathrm{E}}(t)$ and others corresponding to third harmonic generation. Since we are interested only in terms that have the same frequency dependence as the incident light, we ignore the rest.

We obtain

$$
\begin{aligned}
&\left\langle\overrightarrow{\mathrm{P}}^{1}(\omega, t)\right\rangle= \operatorname{Re}\left(\frac{2 N i}{\hbar} \sum_{n} P_{n} \int_{-\infty}^{t} d t_{1} e^{i \omega t_{1}} \operatorname{Im}\left(\left\langle n\left|\vec{\mu}(t) Q\left(t_{1}\right)\right| n\right\rangle\right)\right), \\
&\left\langle\overrightarrow{\mathrm{P}}^{3}(\omega, t)\right\rangle=\operatorname{Re}\left\{\frac { N } { 2 i \hbar ^ { 3 } } \sum _ { n } P _ { n } \left[\int_{-\infty}^{t} d t_{1} \int_{-\infty}^{t_{1}} d t_{2} \int_{-\infty}^{t_{2}} d t_{3}\left(\boldsymbol{\odot}_{++-} e^{i \omega\left(t_{1}+t_{2}-t_{3}\right)}\right) \operatorname{Im}\left(\left\langle n\left|\vec{\mu}(t) Q\left(t_{1}\right) Q\left(t_{2}\right) Q\left(t_{3}\right)\right| n\right\rangle\right)\right.\right. \\
&\left.\left.\quad+\int_{-\infty}^{t} d t_{1} \int_{-\infty}^{t} d t_{2} \int_{-\infty}^{t_{2}} d t_{3}\left(\odot_{++-} e^{i \omega\left(t_{1}+t_{2}-t_{3}\right)}\right) \operatorname{Im}\left(\left\langle n\left|Q\left(t_{3}\right) Q\left(t_{2}\right) \vec{\mu}(t) Q\left(t_{1}\right)\right| n\right\rangle\right)\right]\right\} .
\end{aligned}
$$

Here, $P_{n}$ is the probability that the molecule is in the eigenstate $|n\rangle$ of $H_{0}, \odot_{++-}$is the summation over all distinct permutations in the signs of the terms in the exponent, $Q(t)=\vec{\mu}(t) \cdot \overrightarrow{\mathcal{E}}$ where $\overrightarrow{\mathcal{E}}$ is the constant amplitude of the electric field associated with the incident light, and $\vec{\mu}(t)=\exp \left(i t H_{0} / \hbar\right)$ $\times \vec{\mu} \exp \left(-i t H_{0} / \hbar\right)$. In Eq. (6), the integrations can be carried out and yields the same result as that presented by Ward $^{9}$ if we omit the average over initial states. But we prefer to retain the formula in its present form because it greatly simplifies the model calculations. We also note that Eq. (6) reproduces Wang's ${ }^{8}$ result, and agrees with the result of Armstrong et al..$^{10}$

The linear contribution to $\theta$ from $\left\langle\overrightarrow{\mathrm{P}}^{1}(\omega, t)\right\rangle$ is

$$
\theta=(\pi \omega L / c)\left(\chi_{-,+}^{1}-\chi_{+,-}^{1}\right),
$$

where

$$
\begin{aligned}
\chi_{j k}^{1}= & \frac{N}{\hbar} \sum_{n, n^{\prime}} P_{n} \frac{\omega\left[\left(\mu_{j}\right)_{n n^{\prime}}\left(\mu_{k}\right)_{n^{\prime} n}\right]_{-}}{\omega_{n n^{\prime}}^{2}-\omega^{2}} \\
& +\frac{N}{\hbar} \sum_{n, n^{\circ}} P_{n} \frac{\omega_{n^{\prime} n}\left[\left(\mu_{j}\right)_{n n^{\prime}}\left(\mu_{k}\right)_{n n^{n}}\right]_{+}}{\omega_{n n^{\prime}}^{2}-\omega^{2}} .
\end{aligned}
$$

In Eq. (8), $\omega_{n n^{\prime}}=\left(E_{n}-E_{n^{0}}\right) / \hbar, E_{n}$ is the energy of the molecule in state $n,\left(\mu_{j}\right)_{n n^{\circ}}=\left\langle n\left|\mu_{j}\right| n^{\prime}\right\rangle$, and

$\left[\left(\mu_{j}\right)_{n r^{\prime}}\left(\mu_{k}\right)_{n^{\prime} n}\right]_{ \pm} \equiv\left(\mu_{j}\right)_{n n^{\prime}}\left(\mu_{k}\right)_{n^{\prime} n} \pm\left(\mu_{k}\right)_{n r^{\prime}}\left(\mu_{j}\right)_{n^{\prime} n}$.

We find that $\left[\left(\mu_{j}\right)_{n n^{\prime}}\left(\mu_{k}\right)_{n n_{n}}\right]_{+}$does not contribute to the angle of rotation because it contributes the same for both $\chi_{-,+}^{1}$ and $\chi_{+,-}^{1}$ due to symmetry in $j$ and $k$. The linewidth and level shift caused by the interaction of the isolated atom with its environment have been neglected in the derivation of Eq. (8) [as well as Eqs. (5) and (6)] and will continue to be neglected in the subsequent analysis and discussion.

\section{MODEL CALCULATION}

The application of the Eqs. (5) and (6) to the explicit calculation of the rotation angle of the polarization plane for the general case is not within our reach. To render the explicit calculation tractable and to have both the qualitative and some quantitative features revealed, we consider the following simple model. We assume that all molecules are identical, spinless, noninteracting, and each molecule has only two energy levels. The ground state is a $p$ state, and the excited state is an $s$ state. See the Appendix for some comments regarding the possibility of analysis for more realistic models. We can as well do the calculation for molecules with two energy levels, but having orbital angular momenta opposite to our model. In doing so, the paramagnetic contribution to the rotation angle vanishes. For the purpose of simplicity, we will make the following assumptions: (1) the energy difference between the two levels $\hbar \Omega$ is different from $\hbar \omega$, and (2) the magnetic multiplet width is much smaller than both $\hbar \omega$ and $\hbar(\Omega-\omega)$ and the thermal energy of the medium $\beta \equiv K T$. With these assumptions we obtain two distinct contributions to the Verdet constant for both the lin- 
ear and the nonlinear cases-the paramagnetic part $V_{p}$ and the diamagnetic part $V_{d}$. We get

$$
\begin{aligned}
& V_{p}^{(1)}=\Phi^{(1)}\left(\frac{\omega^{2}}{\Omega^{2}-\omega^{2}}\right) \frac{1}{\beta}, \\
& V_{d}^{(1)}=-\Phi^{(1)} \frac{2 \Omega \omega^{2}}{\hbar\left(\Omega^{2}-\omega^{2}\right)^{2}} ; \\
& V_{p}^{(3)}=\Phi^{(3)} \omega\left(A_{p}+B_{p}\right), \\
& V_{d}^{(3)}=\Phi^{(3)} \omega\left(A_{d}+B_{d}\right) .
\end{aligned}
$$

In these two equations, the superscripts (1) and (3) refer to the linear and the nonlinear contributions respectively, and we find that $\Phi^{(1)}, \Phi^{(3)}$, $A_{p}, B_{p}, A_{d}$, and $B_{d}$ are

$$
\begin{aligned}
& \Phi^{(1)}=\frac{16 \pi^{2} \mu_{B} N e^{2} \Gamma}{9 \sqrt{3} c \hbar}, \\
& \Phi^{(3)}=\Phi^{(1)} \frac{2 \pi^{2} e^{2} \Gamma I}{3 \sqrt{3} c \hbar^{3}} \\
& A_{p}=\frac{4 \hbar \omega\left(3 \Omega^{2}+\omega^{2}\right)}{\beta\left(\Omega^{2}-\omega^{2}\right)^{3}}+\frac{\hbar \omega}{\beta\left(\Omega^{2}-\omega^{2}\right)^{2}}-\frac{2 \hbar^{2} \omega \Omega}{\beta^{2}\left(\Omega^{2}-\omega^{2}\right)^{2}},
\end{aligned}
$$

$$
\begin{aligned}
& B_{p}=\frac{\hbar \Omega^{2}}{\beta \omega\left(\Omega^{2}-\omega^{2}\right)^{2}}, \\
& A_{d}=-\frac{48 \Omega \omega^{3}}{\left(\Omega^{2}-\omega^{2}\right)^{4}}, \\
& B_{d}=-\frac{32 \Omega \omega}{\left(\Omega^{2}-\omega^{2}\right)^{3}},
\end{aligned}
$$

where $\mu_{B}$ is the Bohr magneton, $I$ is the intensity of light, and $\Gamma \equiv\left\langle 0\left\|r Y_{1}\right\| 1\right\rangle\left\langle 1\left\|r Y_{1}\right\| 0\right\rangle$. The quantities $\left\langle 0\left\|r Y_{1}\right\| 1\right\rangle$ and $\left\langle 1\left\|r Y_{1}\right\| 0\right\rangle$ are the reduced matrix elements in the sense of the Wigner-Eckart theorem. We note here that the results for $V_{p}^{(1)}$ and $V_{d}^{(1)}$ are in qualitative agreement with those obtained by Rosenfeld.,4

The above calculation can easily be extended to a two-level system with orbital angular momenta $l \hbar$ and $(l \pm 1) \hbar$ where $l$ is arbitrary. But the complication thereby introduced would not be justified in the context of the present analysis. Again, see the Appendix.

\section{DISCUSSION}

The formulas for the Verdet constants obtained in the previous section are quite complicated. Thus we examine them in two limiting cases: $\Omega \gg \omega$ and $\Omega \ll \omega$, respectively. In the case that $\Omega \gg \omega$, we have

$$
\begin{aligned}
& \frac{V_{p}^{(3)}}{V_{p}^{(1)}}=\alpha_{\mathrm{Nd}} I\left(\frac{\omega_{\mathrm{Nd}}}{\omega}\right)^{2}\left(1-2 \delta \frac{\hbar \omega}{\beta}+14 \delta^{2}\right), \\
& \frac{V_{d}^{(3)}}{V_{d}^{(1)}}=\alpha_{\mathrm{Nd}} I\left(\frac{\omega_{\mathrm{Nd}}}{\omega}\right)^{2}\left(16 \delta^{2}\right)
\end{aligned}
$$

and for the opposite case when $\Omega \ll \omega$, we obtain

$$
\begin{aligned}
& \frac{V_{p}^{(3)}}{V_{p}^{(1)}}=\alpha_{\mathrm{Nd}} I\left(\frac{\omega_{\mathrm{Nd}}}{\omega}\right)^{2}\left(3+\frac{2}{\delta} \frac{\hbar}{\beta}+\frac{18}{\delta^{2}}\right), \\
& \frac{V_{d}^{(3)}}{V_{d}^{(1)}}=32\left(1+\frac{1}{\delta^{2}}\right) \alpha_{\mathrm{Nd}} I\left(\frac{\omega_{\mathrm{Nd}}}{\omega}\right)^{2} .
\end{aligned}
$$

Here, $\omega_{\mathrm{Nd}}$ is the angular frequency of the Nd-glass laser, $\delta \equiv \omega / \Omega$, and $\alpha_{\mathrm{Nd}}$ is given by

$$
\alpha_{\mathrm{Nd}} \equiv \frac{2 \pi^{2}}{3 \sqrt{3}}\left(\frac{e}{\hbar \omega_{\mathrm{Nd}}}\right)^{2} \frac{\Gamma}{c}
$$

From these equations we observe several interesting points. First, these ratios are proportional to $I$. This suggests the possible appearance of nonlinear Faraday effects in high-power laser technology. This also tells us that if the Faraday rotator is used as an isolator in a laser system, the high-intensity portions of the beam will be rotated through different angles than that portion at average intensity, and will tend to be rejected from the beam. Second, the factor $\left(\omega_{\mathrm{Nd}} / \omega\right)^{2}$ implies that these ratios can increase by a few orders of magnitude if long wavelength lasers could be used. For $\mathrm{CO}_{2}$ lasers this factor is 100 . Third, the term containing the quantity $2 \hbar \omega / \beta$ in the paramagnetic ratios will dominate the contribution under the low-temperature condition. At room temperature, this quantity is about 94 for the Nd-glass laser. If we lower the temperature to $1^{\circ} \mathrm{K}$, this quantity will increase to about $2.8 \times 10^{4}$. Fourth, the quantity $\Gamma$ is not amenable to calculation. To give a rough idea of the order of magnitude involved, we approximate $\Gamma$ by $\left(10^{-8} a_{0}\right)^{2} \mathrm{~cm}^{2}$, where $a_{0}$ is the effective ionic radius in $\AA$, to obtain

$$
\alpha_{\mathrm{N} \mathrm{d}} \simeq 0.83 \times 10^{-14} a_{0}^{2} \mathrm{~cm}^{2} / \mathrm{W} .
$$

At this point it should be noted that the intensitydependent modification of Faraday rotation discussed above must be compared with other nonlinear effects influencing polarization rotation. First of all we have stressed the inverse temperature dependence in the ratio, $V_{p}^{(3)} / V_{p}^{(1)}$, in Eq. (18). But there is another contribution to the temperature dependence of paramagnetic Faraday rotation arising from the fact that (in the model used in this paper)

$$
V_{p} \simeq V_{p}^{(1)} \frac{3 \beta}{\mu_{B} B} \frac{\sinh \mu_{B} B / \beta}{1+2 \cosh \mu_{B} B / \beta}+V_{p}^{(3)},
$$

and in the limit $\mu_{B} B / \beta \ll 1$

$$
V_{p} \simeq V_{p}^{(1)}\left(1-\frac{\left(\mu_{B} B / \beta\right)^{2}}{6}+\frac{V_{p}^{(3)}}{V_{p}^{(1)}}\right)
$$

Retaining only the temperature-dependent term in Eq. (18), Eq. (25) becomes 


$$
V_{p} \simeq V_{p}^{(1)}\left[1-\frac{\left(\mu_{B} B / \beta\right)^{2}}{6}-2 \delta \alpha_{N d}\left(\frac{\omega_{N d}}{\omega}\right)^{2} I \frac{\hbar \omega}{\beta}\right] .
$$

Assuming a Nd-glass laser, setting $a_{0}=1$ in Eq. (23), and assigning the value $\delta=0.1$ yields for Eq. (26)

$$
\begin{aligned}
V_{p} \simeq V_{p}^{(1)}[ & 1-8.4 \times 10^{-15}\left(B \frac{T_{0}}{T}\right)^{2} \\
& \left.-7.8 \times 10^{-14} I\left(\frac{T_{0}}{T}\right)\right],
\end{aligned}
$$

where $B$ is in gauss, $T_{0}$ is the standard temperature, and $I$ is the light intensity in $\mathrm{W} / \mathrm{cm}^{2}$. For $T=1^{\circ} \mathrm{K}$, we find that

$$
V_{p} \simeq V_{p}^{(1)}\left(1-7.5 \times 10^{-10} B^{2}-2.3 \times 10^{-11} I\right) .
$$

Thus, at this low temperature, we see that the modification of the temperature dependence of the paramagnetic Verdet constant is dominated by the nonlinear index effect for $I \gtrsim 10^{10} \mathrm{~W} / \mathrm{cm}^{2}$ and $B$ $<10^{4} \mathrm{G}$. For $B>10^{4} \mathrm{G}$, Eq. (25) is no longer valid, and one must use Eq. (24) to calculate $V_{p}$.

Secondly, we note that intensity-dependent rotation of the vibrational ellipse of elliptically polarized laser light can also occur..$^{11}$ This phenomenon is due to the interaction of the light with the medium itself. It has nothing to do with Faraday rotation, i.e., it does not depend upon the presence of an applied magnetic field. The magnitude of this effect can be estimated by use of the formula ${ }^{12}$

$$
\theta_{E} \simeq(6 \pi \omega / n c) \chi_{3}^{1221}\left(E_{-}^{2}-E_{+}^{2}\right) Z,
$$

where $n$ is the linear refractive index of the medium, $Z$ is the path length of light in the medium, $E_{+}$and $E_{-}$are the right and left circularly polarized components of the electric field in the plane perpendicular to the direction of light propagation, and $\chi_{3}^{1221}$ is a quantity to be determined by experiment. Evidently, $100 \%$ perfectly linearly polarized light will not be rotated by this mechanism, but $100 \%$ perfectly linearly polarized light is probably never achievable. The quantity $\theta_{E}$ will be small for $E_{-}^{2} \simeq E_{+}^{2}$ under ordinary experimental conditions. But the Faraday rotation angle contributed by the intensity-dependent term is also not a large quantity. Therefore, it would be interesting to compare these two effects when the light intensity is high. To compute $\theta_{E}$ we need the data for $\chi_{3}^{1221}$ which has been determined experimentally for several liquids ${ }^{13}$ and for some laser glasses..$^{12}$
All these data are measured with ruby laser. But we will assume that $\chi_{3}^{1221}$ does not vary very much for other high-power lasers. Taking $\chi_{3}^{1221}=2.39$ $\times 10^{-15} \mathrm{~cm}^{3} / \mathrm{erg}, n=1.56$ for Owens-Illinois ED-4 glass from Ref. 12 , and setting $\omega=\omega_{\mathrm{Nd}}, Z=1 \mathrm{~cm}$, $I=10^{10} \mathrm{~W} / \mathrm{cm}^{2}$, we find

$$
\theta_{E} \simeq 4.5\left[1-E_{\sim}^{2} / E_{*}^{2}\right] \mathrm{deg} .
$$

Assuming that $E_{-}^{2} / E_{*}^{2} \sim 0.99-0.9999$, then $\theta_{E} \simeq 4.5$ $\times\left(10^{-2}-10^{-4}\right) \mathrm{deg}$. On the other hand, we can estimate the paramagnetic Faraday rotation due to the intensity-dependent term by the formula

$$
\theta_{p}^{(3)} \simeq\left(V_{p}^{(1)} L B\right) \alpha_{\mathrm{Nd}} I\left(\frac{\omega_{\mathrm{Nd}}}{\omega}\right)^{2}\left(1-2 \delta \frac{\hbar \omega}{\beta}\right)
$$

Faraday rotators used as isolators in high-power laser technology are usually designed to rotate the beam by $45^{\circ}$. Thus, we will take $V_{p}^{(1)} L B=45^{\circ}$ in Eq. (31). Setting $I=10^{10} \mathrm{~W} / \mathrm{cm}^{2}, a_{0}=1$, we obtain for Nd-glass laser

$$
\theta_{p}^{(3)} \simeq 3.6 \times 10^{-3}\left(1-2 \delta \frac{\hbar \omega_{\mathrm{Nd}}}{\beta}\right) \mathrm{deg} .
$$

Thus, we see that $\theta_{p}^{(3)}$ can be of the order of, or considerably larger, than $\theta_{E}$.

Finally, we like to stress that the present analysis is based on a simple model. Therefore the quantitative result can only be used for scaling purposes. Further extension of the present analysis could include the effect of spin and the resonance case when $\hbar \omega \simeq \hbar \Omega$. For the former case, Eqs. (5) and (6) are still applicable. For the latter case, Eqs. (5) and (6) must be modified to include linewidth and level shift.

\section{ACKNOWLEDGMENTS}

This work was supported, in part, by KMS Fusion, Inc., Ann Arbor, Michigan. The authors gratefully acknowledge many helpful conversations with Professor John F. Ward.

\section{APPENDIX}

The employment of the simple model in Sec. II for the purpose of obtaining at least some explicit results surely raises some disturbing questions. One is: What does one do if the Faraday-active atoms or ions are to be treated more realistically? Consider the matrix element in the first term of Eq. (6), i.e.,

$$
\begin{aligned}
M_{q} & =\left\langle n\left|\mu_{q}(t) Q\left(t_{1}\right) Q\left(t_{2}\right) Q\left(t_{3}\right)\right| n\right\rangle \\
& =e^{4} \sum_{\substack{n_{1} n_{2} n_{3} \\
q_{1} q_{2} q_{3}}}\left\langle n\left|r_{q}\right| n_{1}\right\rangle\left\langle n_{1}\left|r_{q_{1}}\right| n_{2}\right\rangle\left\langle n_{2}\left|r_{q_{2}}\right| n_{3}\right\rangle\left\langle n_{3}\left|r_{q_{3}}\right| n\right\rangle e^{i \omega_{n n_{1}} t+i \omega_{n_{1} n_{2}} t_{1+i \omega_{n_{2}} n_{3}} t_{2}+i \omega_{n_{3} n} t_{3} \mathcal{E}_{q_{1}}^{*} \mathcal{E}_{q_{2}}^{*} \mathcal{E}_{q_{3}}^{*} .}
\end{aligned}
$$


Without considerable knowledge of the eigenstates, $|n\rangle$, little further veduction of this expression is possible. In fact, as mentioned in the text, the best that we can do in this circumstance is insert the expression in Eq. (A1) - and a similar one for the matrix element in the second term-in Eq. (6), sum over the indicated permutations and carry out the time integrals; thereby reproducing formal results presented elsewhere, e.g., as given by Ward in Ref. 9. Even if we assume that the total angular momentum of the states, $|n\rangle$, is characterized by definite values of $l$ and $m$ (orbital angular momentum and projection along the magnetic field, respectively) only trivial further reduction. is possible, i.e.,

$$
\left\langle l_{1} m_{1}\left|r_{q}\right| l_{2} m_{2}\right\rangle=\left(l_{1} 1 m_{1} 2 \mid l_{1} 1 l_{2} m_{2}\right) \cdot\left\langle l_{1}\left\|r Y_{1}\right\| l_{2}\right\rangle
$$

where the first factor is a Clebsch-Gordan coefficient according to the convention of Condon and Shortley ${ }^{14}$ and the second factor is the reduced matrix element. The formula in Eq. (A1) remains unwieldy and is probably not subject to evaluation since the reduced matrix elements are generally not calculable. Thus, it seems to us that analysis of the intensity-dependent contribution to Faraday rotation in the realistic case of multilevel atoms or ions is presently out of reach. An experiment to try to observe the phenomenon seems more in order.

Another question raised by our choice of a model is: How realistic is it to assume $p$ and $s$ states for the ground and single excited states, respectively? The answer, of course, is that the choice is not realistic at all. Faraday rotators used in highpower laser technology are usually glass, doped with paramagnetic, rare-earth ions-all characterized by large values of the orbital angular momentum in the ground state. However, we are stymied here also even if we resort to a two-state model calculation. Although it is fairly conventional to label these ionic states according to Rus.sell-Saunders spectroscopic notation, they are not pure Russell-Saunders states; but rather linear superpositions thereof with the labeling $l$-value dominant. Thus, a "realistic" calculation to reveal the $l$ dependence for a two-state model ion is still too complicated to be warranted by the present investigation. However, if we do assume a ground state of definite $l$ and a single excited state of definite $l-1$ say, an illustrative reduction of Eqs. (5) and (6) can be achieved for paramagnetic rotators, ${ }^{7}$ and may be of some interest. The point is that, in this case, the energy differences in the time-dependent exponentials may be considered to be independent of the magnetic quantum numbers so that the sum over these quantum numbers can be explicitly carried out to yield

$$
\begin{aligned}
& V_{p}^{(1)}=\Phi^{(1)}(l, l-1) \frac{\omega^{2}}{\Omega^{2}-\omega^{2}} \frac{1}{\beta} \frac{(l+1)[(2 l-1)(2 l+1)]^{1 / 2}}{2} \\
& V_{p}^{(3)}=I \Gamma(l, l-1) \frac{\pi^{2} e^{2}}{c \hbar^{2}} \Phi^{(1)}(l, l-1) \frac{\omega}{\beta}\left(\frac{(l+1)(2 l+3)}{15 l} \frac{\Omega^{2}+\omega^{2}}{\omega\left(\Omega^{2}-\omega^{2}\right)^{2}}-\frac{2(l+1)(2 l-1)(4 l+1)}{15 l} \frac{\hbar \omega \Omega}{\beta\left(\Omega^{2}-\omega^{2}\right)^{2}}\right. \\
&+\frac{2(l+1)(2 l-1)(4 l+1)+4\left(6 l^{2}-1\right)}{15 l} \frac{\omega\left(3 \Omega^{2}+\omega^{2}\right)}{\left(\Omega^{2}-\omega^{2}\right)^{3}} \\
&\left.+\frac{2(l-1)(2 l+3)}{15 l} \frac{\omega}{\left(\Omega^{2}-\omega^{2}\right)^{2}}\right)
\end{aligned}
$$

where

$$
\begin{aligned}
& \Phi^{(1)}(l, l-1)=\frac{16 \pi^{2} e^{2} \mu_{\beta} N \Gamma(l, l-1)}{9 c \hbar(2 l+1)}, \\
& \Gamma(l, l-1)=\left\langle l\left\|_{r} Y_{1}\right\| l-1\right\rangle\left\langle l-1\left\|r Y_{1}\right\| l\right\rangle,
\end{aligned}
$$

and

$$
\Omega_{i}=\left(E_{1}-E_{2}\right) / \hbar .
$$

For $l=1$ Eqs. (A2) and (A3) reduce to the results presented in the text for the simple model.

The explicit dependence upon the orbital angular momentum, $l$, can be quite misleading because the reduced matrix elements [Eq. (A5)] can be sensitive function of $l$. Thus here again we are forced to conclude that further analysis is probably unilluminating $^{15}$ until informed by some experimental observations of nonlinear Faraday rotation.
*This work is drawn in part from a thesis submitted as partial fulfillment of the requirements for the $\mathrm{PhD}$ degree in Nuclear Engineering at the University of Michigan.

\footnotetext{
${ }^{1}$ A. Sommerfeld, Optics (Academic, New York, 1964). ${ }^{2} \mathrm{~J} . \mathrm{H}$. Van Vleck, The Theory of Electric and Magnetic Susceptibilities (Oxford U.P., London, 1932).

${ }^{3} \mathrm{G}$. Charatis et al., in Proceedings of the Fifth Confer-
} 
ence on Plasma Physics and Controlled Nuclear Fusion Research, Tokyo, 1974 (International Atomic Energy Agency, Vienna, 1975), paper CN-33/F1.

${ }^{4}$ Laser Program Annual Report-1974, UCRL-50021-74 (unpublished).

${ }^{5}$ L. Rosenfeld, Z. Phys. 57, 835 (1930).

${ }^{6}$ R. Serber, Phys. Rev. $\underline{41}, 489$ (1932).

${ }^{7} \mathrm{~J} . \mathrm{H}$. Van Vleck and M. H. Hebb, Phys. Rev. $\underline{46}, 17$ (1934).

${ }^{8}$ C. C. Wang, Phys. Rev. B 2 , 2045 (1970).

${ }^{9}$ J. F. Ward, Rev. Mod. Phys. 37, 1 (1965).
${ }^{10} \mathrm{~J}$. A. A rmstrong, N. Bloembergen, J. Ducuing, and P. S. Pershan, Phys. Rev. 127, 1918 (1962).

${ }^{11}$ P. D. Maker and R. W. Terhune, Phys. Rev. 137, A801 (1965).

${ }^{12}$ Adelbert Owyoung, IEEE J. Quantum Electron. QE9, 1064 (1973).

${ }^{13}$ P. D. Maker et al ., Phys. Rev. Lett. 12, 507 (1964).

${ }^{14}$ E. V. Condon and G. H. Shortley, The Theory of Atomic Spectra (Cambridge U.P., London, 1963).

${ }^{15}$ B. R. Judd, Phys. Rev. 127, 750 (1962). 